\section{Model crystals}

\section{Penny plain, tuppence coloured}

from Robert W. Cahn

A FLOURISHING community of theoreticians seeks to clarify processes in crystals - phase transformations, critical phenomena, radiation damage, melting and vitrification - by simulating them in powerful computers. The latest attempts to model crystals realistically are reported in two papers elsewhere in this issue ${ }^{1.2}$.

Physical modelling of crystals began in the late 1940 s with the two-dimensional soap-bubble raft of Bragg, Nye and Lomer. Given the right bubble radius, such rafts reproduced well the interatomic force law typical for metals, and were used to simulate the motion of dislocations under shear stress. They were also used to model metallic glasses, but for this purpose, two populations of bubbles of different radii were essential to inhibit 'crystallization's. Such rafts have been well used, notably by Argon ${ }^{4}$, to interpret mechanical properties of metallic glasses.

In the 1960s, Bernal produced his influential liquid model with monosized steel balls kneaded in a football bladder, which was later extended to imitate metallic glass structures. In contrast to the bubble raft, Bernal's model suffered from the fact that the balls were hard and could not imitate the 'soft' interatomic force laws appropriate for alloys: this inadequacy was overcome, both literally and metaphorically, by the introduction of software to allow relaxation of a Bernal model via computer simulation.

More recently, a new family of 'macroparticulate' crystals has appeared in the form of colloidal crystals ${ }^{5}$, regular assemblies of equal submicron-sized spheres, typically of a polymer or of silica. These crystals were not intended as models: their study grew from observations of natural macroparticulate crystals - the crystalline viruses and opal ${ }^{6}$ (which consists of regular arrays of monosized amorphous silica spheres). The first artificial macroparticulate crystals to be made, gemquality opals, were made by gravityinduced sedimentation of colloidal silica suspensions by Pierre Gilson at his factory in northern France ${ }^{7}$ and in commercial production since 1974 . How these opals are stabilized after sedimentation (sintering or low-temperature adhesion) is a commercial secret.

The study of silica (and polymeric) macroparticulate crystals has accelerated rapidly since then (although it is doubtful whether many of the physicists concerned know of Gilson's early triumph). The use of macroparticulate crystal assemblies of ceramic microspheres as 'green' precursors for sintering has now been proposed and a promising start has been made with silica $^{8}$ (as discussed in these columns ${ }^{9}$ ).

One of the papers in this issue ${ }^{1}$ follows the colloidal approach to crystal simulation: Pusey and van Megen used monosized polymethylmethacrylate spheres, $0.3 \mu \mathrm{m}$ in diameter and stabilized in suspension by a thin coating of another polymer. The organic suspensions were of high volume fractions, ranging from 0.39 to 0.53 . After mechanical homogenization, each suspension was allowed to settle. In the volume fraction range $0.44-0.50$, polycrystalline structures have bright opalescence; suspensions below 0.41 do not crystallize at all; whereas in the range $0.41-0.44$, a stable liquid-plus-crystal two-phase state forms. But the really novel feature occurs in volume fractions above 0.50 , where the viscous suspension remains in a 'glassy' form indefinitely: in effect, this is a kinetically metastable random dispersion which is too dense, and therefore too immobile, to order. Unlike two-dimensional bubble rafts, therefore, in three dimensions it is possible to create a glass from a population of monosized spheres. (Whether this is possible depends on the mobility of the units; be they macroparticles or atoms, pure metals cannot be prevented from crystallizing even at cooling rates in excess of $10^{12} \mathrm{~K} \mathrm{~s}^{-1}$.) Pusey and van Megen's method could be used to make a form of artificial opal if a way to stabilize the crystals against accidental randomization can be found.

Pusey and van Megen's suspensions differ radically from another kind which also show unexpected properties: Clark and Ackerson ${ }^{10,11}$ demonstrated that very dilute ( 0.1 per cent) suspensions of highly charged polymer spheres can 'crystallize' into body-centred cubic crystals while remaining suspended, but randomize (melt) after vigorous shear deformation - an observation which could shed light on mechanical instability models of melting for ordinary atomic crystals.

The other paper in this issue, by Georges et al. $^{2}$, exploits bubble rafts to simulate, in two dimensions, a microhardness indentation test. A conical indenter is simulated by a suitably shaped (poly)crystalline raft; the test object is a large monosized polycrystalline raft covered by an amorphous 'coating' consisting of a mix of two bubble sizes. The authors do not explain why they chose to test a glass-coated crystalline-simulated sample, but in the light of some recent tests on real glassy wear-resistant coatings, it was a happy choice. The simulated coating proves extremely resistant to penetration, but its behaviour is intimately affected by its crystalline substrate, which undergoes a largely elastic deformation. On unloading, this elastic distortion recovers and the form of the indentation recovers with it, so much so that the end-result is a small mound instead of an indentation.

This model system with its properties is of considerable interest in relation to recent work on the use of an ultramicrohardness tester, the nanoindenter, invented and named by W.C. Oliver ${ }^{12.13}$. This instrument is extremely sensitive, using measurements of the vertical displacement of the indenter (as small as 20 $\mathrm{nm}$ ) to evaluate the residual indentation. (Direct measurement of such minute features would require scanning electron microscopy.) The instrument allows the increase in effective hardness for indents less than $100 \mathrm{~nm}$ deep to be confirmed and interpreted $^{12}$. It was also found that considerable elastic recovery of the indentation takes place, and that this is essentially caused by a recovery of the 'hinterland' of the indentation, just as in the bubble simulation. But nanoindentations in real crystals do not invert and finish up as mounds.

Oliver and his collaborators have also studied the nanohardness of coated (ionimplanted) metals ${ }^{13}$. Of relevance to the present discussion is the behaviour of steel ion-implanted with both titanium (Ti) and carbon (C). If the concentrations of both are sufficient, the coating is very hard and wear-resistant. Such Ti/C implants are known to be amorphous, and the excellent mechanical properties of the layers are directly correlated with their vitreous nature. Georges et al. ${ }^{2}$ agree with these findings in the sense that their amorphous simulated coating proved impenetrable, being always pushed ahead of the moving indenter, but disagree in the sense that the yield stress they observed for the coating was lower than that for the crystalline substrate. Amorphous $\mathrm{Ti} / \mathrm{C}$ coatings are stronger, harder and more wear-resistant than the underlying steel.

1. Pusey, P.N. \& van Megen, W. Nature 320, 340 (1986)

. Georges, J.M., Meille, G., Loubet, J.L. \& Tolen, A.M Nature 320, 342 (1986)

3. Simpson, A.W. \& Hodkinson, P. Nature 337, 320 (1972)

4. Argon, A.S. \& Kuo, H.Y. Mater. Sci. Engng. 39, 101 (1979)

. Pieranski, P. Contemp. Phys. 24, 25 (1983)

6. Darragh, P.J., Gaskin, A.J. \& Sanders, J.V. Scient. Am. 234 (4), 84 (1976)

Anderson, B.W. Gem Testing 9th edn (Butterworth, Lon don, 1980).

8. Sacks, M.D. \& Tseng, T.-Y. J. Am. Ceram. Soc. 67, 526 (1984)

Calvert. P.D. Nature News and Views 317, 201 (1985)

10. Clark, N.A., Hurd, A.J. \& Ackerson, B.J. Nature 281. 57 (1979).

11. Ackerson, B.J. \& Clark, N.A. Phys. Rev. Lett. 46, 123 (1981)

12. Pethica, J.B., Hutchings, R. \& Oliver, W.C. Phil. Mag. A48. 593 (1983)

13. Pethica, J.B., Hutchings. R. \& Oliver, W.C. Nucl. In strum. Meth. 209/210. 995 (1983)

Robert W. Cahn is currently Fairchild Distinguished Scholar in the Keck Engineering Laboratories, California Insitute of Technology, Pasadena, California 91125, USA. 